\title{
Respuesta de la comunidad fitoplanctónica de una laguna ecuatorial hipereutrófica a la introducción de un nuevo ecodemo
}

\author{
John Jairo Ramírez R. \\ Universidad de Antioquia, Instituto de Biología. Apartado 1226. Medellín, Colombia. \\ E-mail: johnra77@yahoo.com
}

\section{RESUMEN}

Se analiza el efecto de la introducción accidental del ecodemo Botryococcus braunii en la estructura de la comunidad fitoplanctónica de la laguna del Parque Norte, un cuerpo de agua raso e hipereutrófico tropical. ¿Cuál fue este efecto, si la comunidad fitoplanctónica en esta laguna siempre presentó una composición de especies pobre y supuestamente estable frente a tensores de diferente índole? Se prevé que, si como respuesta a la introducción la comunidad aumenta el orden y sustituye al dominante competitivo, entonces el ecodemo introducido constituye una entidad disipativa de la inestabilidad generada por su introducción y de la ya presente en el sistema. ¿Ocurrió esto? Efectivamente, pues la introducción generó una interacción asimétrica con las especies residentes y se constituyó en una fuente de entropía para los ecodemos dominantes actuales. Dado que el ecodemo introducido fue la única especie capaz de integrar adecuadamente la adquisición de energía y su acumulación, de oscilar menos dentro del sistema y de su comunidad y la única especie entre las presentes, en que las características de estructura disipativa eran más claramente reconocibles, ocurrió la exclusión de los antiguos dominantes. Estos pertenecían principalmente a las cianoprocariotas, entre los que destacaba Microcystis aeruginosa. Un dominante competitivo fue sustituido por otro más estable y adaptado. B. braunii puede entonces ser considerado una estructura disipativa, pues muestra un alto grado de uniformidad en tamaño, una talla relativamente grande, ciclo de vida largo, alto grado de continuidad (expresado a través de la dominancia en el sistema), mantenimiento de la máxima biomasa en relación a la entrada de energía y una menor oscilación de la misma al superar las pérdidas inducidas por diferentes factores.

Palabras clave: laguna eutrófica tropical, fitoplancton, introducción accidental, entropía, disipación.

\begin{abstract}
The effect of the accidental introduction of the ecodeme Botrycoccus braunii on the structure of the phytoplankton community of the Parque Norte lagoon is analyzed. This is a tropical, shallow and hypereutrophic body of water. If the phytoplankton community of this lagoon always presented a poor species composition supposedly stable enough to face different kinds of tensors, what was the effect of the introduction on the phytoplankton community? If the response is that the community increases the order and substitutes the competitive dominant, then the introduced ecodeme constitutes a dissipative entity of the instability generated by its introduction and of the system's already present instability. Has such thing occurred? Indeed, because the introduction generated an asymmetric interaction with the resident species, and because this interaction constituted a source of entropy for the dominant's resident ecodemes. Since the introduced ecodeme was the unique species able to integrate the acquisition and accumulation of energy, showed few oscillations within the system and its community and it was the unique species among the already resident species of the system that presented more clearly recognizable dissipative structure characteristics, this situation provoked the exclusion of the former dominants. These belonged mainly to cianoprokarionts among which Microcystis aeruginosa was important. A competitive dominantwas substituted by another more stable and adapted one. B. braunii can be then considered a dissipative structure because it showed a high uniformity in size, a relatively large size, a long life cycle, high continuity as expressed through its dominance in the system), maintaining of the maximum biomass in relation to the energy income, and a smaller biomass oscillation by surpassing the induced loss by different factors.
\end{abstract}

Keywords: eutrophic tropical lagoon, phytoplankton, accidental introduction, entropy, dissipation. 


\section{INTRODUCCIÓN}

Un lago es un sistema dinámico conformado por ecodemos interactuantes cuya finalidad es alcanzar un estado ordenado (uniformidad) y de baja producción de entropía, alta exergía y resiliencia (Johnson, 1981; Reynolds, 1997). A pesar de la capacidad de los sistema lénticos para organizarse a sí mismos, ninguno de ellos alcanza un estado de equilibrio reconocible, pues en general, por su condición de sistemas abiertos, se rigen por los principios de los sistemas de no equilibrio, donde el efecto de las perturbaciones de uno u otro tipo, desempeña un importante papel. Puede decirse entonces que los procesos de organización de la comunidad están fuertemente influenciados por fuerzas externas y que, bajo su acción, las comunidades retroceden a menudo a estados más primitivos de organización, pues se destruye momentáneamente parte de la exergía ganada en un espacio-tiempo determinado. Dicha destrucción ocurre para ajustarse a las prescripciones de la segunda ley de la termodinámica, esto es, disipar los gradientes de energía y/o materia "introducidos" por las perturbaciones de carácter biótico o abiótico.

Como sistemas termodinámicos abiertos, los sistemas ecológicos deben obedecer la segunda ley en una de dos maneras: 1) deben disipar alta entropía de materia y energía desde sí mismos hacia sus ambientes, lo que los ajustará a la idealización de las estructuras disipativas; o 2) deben exhibir crecimiento espontáneo a través del tiempo. En este caso, se ajustarían a la idealización de la fase espacial expandiente. Los sistemas ecológicos exhiben ambas clases de conducta (Brooks \& Wiley, 1988).

Consecuentemente, los sistemas ecológicos exhiben un número de características de los sistemas termodinámicos que están lejos de la entropía máxima presente en los sistemas cerrados: muchas de sus conductas son irreversibles y la dinámica de tales conductas contiene un elemento estocástico probabilístico; están ligados a sus entornos por intercambios de energía que permiten el mantenimiento de una estructura organizada, y se hallan lejos de la máxima entropía en términos de intercambio de materia, debido a que los productos finales son eliminados desde o transportados a otras parte del sistema (Brooks \& Wiley, 1988).

La función de disipación en un sistema ecológico está conformada por una función de disipación externa y una función de disipación límite. Esta última tiene dos componentes: uno pertinente a la acumulación de biomasa independiente de los cambios en información genética, y el otro pertinente a la acumulación de información genética independiente de las consideraciones de biomasa. El funcionamiento biológico de cualquier clase envuelve los tres componentes. Sin embargo, dependiendo de la escala de tiempo escogida, los procesos biológicos pueden aparecer dominados por uno de ellos. Durante los intervalos cortos de tiempo, predomina la función de disipación externa. Los sistemas biológicos vistos desde esta perspectiva temporal (tiempo fisiológico) deben conformarse a la idealización de las estructuras disipativas, ya que la conducta está dominada por la disipación desde el sistema. Cuando visto en escalas intermedias de tiempo, predomina la acumulación de biomasa. Esto pertenece a una variedad de procesos biológicos que incluyen el desarrollo (tiempo ontogenético), la reproducción (tiempo generacional), y la sucesión de la comunidad y el turnover (tiempo ecológico). Finalmente, los procesos biológicos operando en intervalos de tiempo mayores (tiempo evolutivo) son dominados por la acumulación de información genética dentro del sistema por el componente biótico del mismo (Brooks \& Wiley, 1988).

La estabilidad es la capacidad de un sistema de retornar al estado basal después de una perturbación por un agente externo. El grado de estabilidad se caracteriza por la amplitud de la desviación a partir del estado inicial, la rapidez de la respuesta del sistema y la velocidad para amortiguar la desviación del estado basal inicial. Si el sistema es sometido a pequeños disturbios, y consigue resistirlos retornando a su estado basal o a algo próximo a él, se dice que el sistema es localmente estable. Pero los sistemas ecológicos están sujetos a perturbaciones de 
mayor intensidad, lo cual requiere introducir un segundo tipo de estabilidad: la estabilidad global. Una región de estabilidad local presentará estabilidad global si el sistema después de grandes perturbaciones y una vez cesada la perturbación, regresa al mismo estado. No siempre ocurre esto último y uno de los problemas de la ecología es delimitar la estabilidad global para diversas comunidades (Krebs, 1985; Begon et al., 1990). Las comunidades estables son, por definición, aquellas capaces de persistir de la forma que son (Pimm, 1984), debido a las altas coherencia y resiliencia de que hacen gala.

La estabilidad local de un sistema se prueba entonces aplicando pequeños disturbios y observando el resultado. Si el resultado obtenido difiere marcadamente de aquel que se presentaba cuando no había disturbio, se dice que el sistema es inestable y el efecto sobre las estructuras disipativas del sistema es notable, pues se dice que no "superaron" la entropía alóctona incorporada por la perturbación. Si el efecto del disturbio actual desaparece con el tiempo y la respuesta es similar, perturbado o no, se dice que el sistema es asintóticamente estable (Cunningham, 1963). La perturbación puede estar representada por factores individuales o por una asociación de ellos; en este trabajo está encarnada en la introducción de ecodemos extraños al sistema. Sin embargo, y en concordancia con Prigogine (1997), la concepción más apropiada de perturbación está ligada al concepto de inestabilidad, atado a su vez a la noción de caos y de probabilidad, tan opuestos a las de reversibilidad, determinismo y equilibrio presentes en la ecología y la limnología clásicas. La fuerza de estas concepciones tradicionales ha llevado a desconocer, más apropiadamente a olvidar, que las pequeñas perturbaciones o inestabilidades se amplifican y llevan a que unas trayectorias inicialmente cercanas se separen y se "bifurquen" siguiendo nuevos caminos. Estas son las estructuras de no equilibrio o estructuras disipativas caracterizadas por el concepto de irreversibilidad y las rupturas de simetría. Estas estructuras de no equilibrio sólo existen mientras el sistema disipa energía y permanece en interacción con el exterior. Contrastan con las de equilibrio, como los cristales, que una vez formados, pueden permanecer aislados y son estructuras muertas, sin disipación de energía. La inestabilidad, el caos, la no causalidad nos obliga entonces a acercarnos, hoy, a un esquema probabilista más real (Prigogine, 1997).

\section{ANTECEDENTES}

\section{Caracterización física y química}

La Laguna del Parque Norte (LPN; $06^{\circ} 17^{\prime} \mathrm{N}-$ $75^{\circ} 33.4^{\prime} \mathrm{W}$ ) es un sistema raso con algunas irregularidades en el contorno $\left(\mathrm{D}_{\mathrm{L}}=1.31\right)$, pendientes fuertes en las orillas, fondo plano, zona litoral pobremente desarrollada $\left(\mathrm{D}_{\mathrm{v}}=2.29\right)$ y alta inestabilidad en la columna de agua; por ello, se clasifica como un sistema polimíctico cálido continuo, que cede calor por convección al ambiente (Ramírez, 2000a). El mayor cambio perceptible en el ambiente físico posterior a la introducción fue el aumento en la penetración de luz que pasó de $0.20-0.30 \mathrm{~m}$ a toda la columna de agua (aproximadamente $1.60 \mathrm{~m}$ ). A pesar de los cambios estructurales de la comunidad planctónica, variables ambientales como alcalinidad, conductividad eléctrica y $\mathrm{pH}$ han presentado siempre valores altos y poco variables, lo que muestra un sistema altamente tamponado, con una reserva alcalina considerable y poco dinámico desde el punto de vista de las variables mencionadas. Las altas conductividades (2000$2700 \mu \mathrm{S} / \mathrm{cm}, \mathrm{CV}=12 \%)$, alcalinidades $(240-250$ $\mathrm{mgCaCO}_{3} / \mathrm{l}, \mathrm{CV}=11.4 \%$ ) y dureza (total $=101$ $103 \mathrm{mgCaCO}_{3} / \mathrm{l}, \mathrm{CV}=5.5 \%$ ) indican aguas de composición iónica rica, cuyos valores son debidos básicamente a las altas concentraciones de calcio, magnesio, cloruros $(480-750 \mathrm{mgCl} / 1$, $\mathrm{CV}=10 \%)$ y material disuelto en general (Ramírez \& Díaz, 1994; Sánchez \& Ramírez, 2000; Ramírez \& Alcaráz, 2002). Este último se refleja en los valores medios de los sólidos totales disueltos (1187. $2 \mathrm{mg} / \mathrm{l})$, los cuales corroboran que se trata de aguas altamente mineralizadas (Noreña \& Ramírez, 2001). El pH en todas 
las colectas presentó valores básicos y poco variables $(8.4-8.6, \mathrm{CV}=4 \%)$ debidos a la alcalinidad y conductividad altas.

\section{Caracterización biológica}

La LPN desde las primeras investigaciones (Ramírez, 1987) presentó densidades fitoplanctónicas altas y las algas del orden Chlorococcales fueron dominantes, estando representadas especialmente por Keratococcus bicaudatus; el valor de riqueza hallada en este año fue de 37 taxones, para una diversidad de 2.03 nats/ind y una equidad de 0.56. En un estudio efectuado dos años más tarde se observó como especies dominantes a Scenedesmus quadricauda y a Tetraedron minimum, y se obtuvo evidencia de blooms de Microcystis cf. aeruginosa, Aphanizomenon aphanizomenoides (Ramírez, 2000b) y Anabaenopsis (Ramírez, 1994); hubo además desaparición de algunas especies importantes en la primera investigación (principalmente Crucigenia tetrapedia). Para este periodo la riqueza disminuyó a 20 taxones, la diversidad a 1.49 nats y la equidad a 0.49 . La biomasa media, estimada a partir de la clorofila $a$ fue de $37.96 \mathrm{mg} / \mathrm{m}^{3}$ (Ramírez \& Díaz, 1994).

En 1991 fue introducida accidentalmente el alga verde Botryococcus braunii Kützing 1849, proveniente de un embalse oligo-mesotrófico situado a 2100 m.s.n.m, la represa La Fé. En un nuevo trabajo realizado en 1999 Corbacho encontró que $B$. braunii era la especie dominante (densidad media $=108000 \mathrm{ind} / \mathrm{l})$, acompañada por otras dos especies del mismo género: B. terribilis y Botryococcus sp. Como consecuencia de lo anterior, desaparecieron la mayoría de las clorococales anteriormente halladas, no se registraron más blooms de Microcystis ni otras cianoprocariotas y comenzaron a aparecer nuevos organismos en el fitoplancton, entre ellos algunas diatomeas (especialmente Cyclotella meneghiniana) y euglenofitas (Euglena oxyuris principalmente). La biomasa hallada durante este estudio $\left(139.3 \mathrm{mg} / \mathrm{m}^{3}\right)$ fue 3.7 veces mayor que la de 1989. Si se acepta que sistemas con valores promedios de clorofila iguales o superiores a $100 \mathrm{mg} / \mathrm{m}^{3}$ son ambientes hipertróficos (Alvarez Cobelas \& Jacobsen, 1992), los valores encontrados tipifican la LPN como un sistema de este tipo. Paralelamente a la introducción de $B$. braunii, se introdujo el copépodo calanoide Arctodiaptomus dorsalis, el cual desplazó al copépodo ciclopoide Thermocyclops decipiens, codominante en la comunidad zooplanctónica con el rotífero Brachionus plicatilis (Ramírez \& Díaz, 1996-1997), quien ahora alterna la dominancia con $A$. dorsalis.

¿Por qué la comunidad fitoplanctónica de la LPN responde de esta manera a la "introducción" de una nueva estructura disipativa, representada por B. braunii, si siempre presentó una composición de especies pobre y supuestamente estable que implica una mayor conectividad y por tanto una mayor estabilidad, persistencia, resiliencia y resistencia al cambio?

\section{DISCUSIÓN}

Antes de comenzar, vale la pena destacar que dada la pequeña escala de los taxones del plancton, los fenómenos de la introducción de especies y sus consecuencias raramente son tenidos en cuenta en esta biocenosis; no en tanto, como se muestra en este artículo, el resultado es similar aunque poco documentado; en Colombia, por lo menos, jamás se ha publicado nada al respecto. La mayoría de los estudios referentes a este fenómeno en los sistemas acuáticos se han llevado a cabo en peces y en macrófitas. En ecosistemas acuáticos es bien conocido el trabajo de Zaret \& Paine (1973) quienes documentaron la introducción de Cichla ocellaris, un pez cíclido nativo del río Amazonas, en el lago Gatún en Panamá. Esta especie asumió rápidamente la dominancia y redujo drásticamente la diversidad de la fauna autóctona. Según Ros (1991) en un porcentaje superior al $70 \%$ de los casos estudiados, las especies exóticas de peces ocasionan el declive de la ictiofauna nativa. La introducción de especies ícticas ha ofrecido evidencia empírica a la teoría del control top-down en los ecosistemas acuáticos. Sin embargo, la eviden- 
cia registrada en la presente investigación apoya la teoría del control bottom-up, es decir, aquel que se ejerce desde los productores del sistema.

Cada sistema es un conjunto de diversos elementos, compartimientos o unidades, cada uno de los cuales puede existir en muchos estados diferentes, de manera que la selección de un estado está influenciada por los estados de los otros componentes del sistema, de los cuales formarán parte los componentes introducidos. Puede considerarse entonces que cualquier elemento de este conjunto que pueda adoptar distintos estados, automáticamente permanecerá, después de algún tiempo en el más estable de aquellos.

$B$. braunii, así como A. dorsalis, son ecodemos, estructuras disipativas que compiten con las demás para incrementar la adquisición de energía desde el entorno y conservarla incrementando su biomasa y su número de individuos. Toda estructura disipativa tiende hacia un estado estable (aquel de menor producción de entropía) y al alcanzarlo desplazará a otros ecodemos menos efectivos en la disipación de la entropía introducida al sistema por las nuevas perturbaciones a las que se ve sometido. Un ecodemo como grupo organizado pretende alcanzar un orden (la uniformidad) y llegar al estado de mínima producción de entropía; a ambas condiciones se llega cuando el ecodemo se constituye en el conjunto dominante. En primera instancia, estos dos ecodemos parecen no interactuar. Según Johnson (1981), una especie heterotrófica, como A. dorsalis, tiende a inducir un incremento en el flujo de energía y a intensificar la fluctuación en las especies de que se alimenta. Vistas de esta manera, estas fluctuaciones pudieron favorecer las condiciones para que B. braunii ampliara su nicho y alcanzara la condición de ecodemo dominante actual en el sistema. Vale la pena recordar que, según los postulados de la ecología teórica, el éxito alcanzado por una especie introducida está determinado por el binomio colonización-extinción (nicho vacío-nicho ocupado, nicho fundamental-nicho realizado y cambio ontogenético del nicho).

Según los postulados termodinámicos, cualquier interacción entre sistemas o subistemas incrementa la entropía, lo cual va en contra de la meta individual de disminuir la producción de la misma. La interacción asimétrica entre la especie fitoplanctónica introducida y las residentes constituye pues una fuente de entropía. La alternativa para disminuir su incremento sería entonces evitar la interacción, lo cual, en el sistema LPN es bastante improbable, primero por la condición polimíctica del sistema, y segundo por la invasión de las fronteras del nicho de las especies residentes dominantes por la introducida. Como consecuencia de la interacción desigual ocurre una exclusión real de los antiguos dominantes. El nuevo dominante es la única especie capaz de integrar adecuadamente la adquisición de energía y su acumulación, siendo la que oscila menos dentro del sistema y de su comunidad, la más cercana al estado estable y la única en que las características de estructura disipativa son más claramente reconocibles (Johnson, 1981). B. braunii posee las características necesarias para ser considerado como tal, pues muestra un alto grado de uniformidad en tamaño, una talla relativamente grande, ciclo de vida largo, alto grado de continuidad (expresado a través de la dominancia en el sistema), mantenimiento de la máxima biomasa en relación a la entrada de energía y una menor oscilación de la misma al superar las pérdidas inducidas por diferentes factores. Todas estas características corresponden a un estratega del tipo S (Ramírez \& Corbacho, 2004). Las dos últimas propiedades demuestran que la resiliencia y coherencia de la especie son altas. Vale la pena resaltar que los estrategas $\mathrm{S}$ son más resistentes y persistentes frente a las perturbaciones, pero si la inestabilidad generada es frecuente e intensa, tendrán poca capacidad de recuperación (resiliencia y elasticidad bajas) cuando comparados con los estrategas $\mathrm{C}$ que tienen poca resistencia a las inestabilidades, pero alta resiliencia debido a sus altos tiempos de renovación (Begon et al., 1990).

Un cuerpo de agua léntico puede considerarse, en cierta medida, como una isla que responde a las condiciones estipuladas en la teoría de la Biogeografía Insular. Esta teoría postula que la introducción de una especie puede conducir a 
la desaparición de una o varias especies, con o sin efecto en el nicho de las especies nativas; desaparición de una o varias especies por alteración de la cadena trófica del sistema; y finalmente, al establecimiento de la especie invasora debido a su capacidad para convertirse en una estructura disipativa adecuada. A todas luces, lo sucedido en la LPN se ajusta a la segunda y tercera consideración.

Se reconoce que una perturbación o inestabilidad ha ocurrido determinando la calidad de la respuesta de las especies de alto nivel en el sistema; esta respuesta se juzga por la resiliencia y la persistencia estructurales de la comunidad frente a la severidad de la instabilidad, y se califica en términos bien sea de una alteración de la biomasa total o de su redistribución entre las especies presentes (Reynolds, 1997). Si la primera es muy débil o la última muy fuerte, la sucesión continuará su ruta normal y se puede concluir que la comunidad "asimiló" el impacto y mantuvo su estructura. En general, y debido al efecto de las perturbaciones, lo que se observa son una serie de episodios sucesionales que avanzan hasta que ocurra un evento de magnitud suficiente que demore, detenga o desmantele abruptamente el desarrollo sucesional corriente. En nuestro caso, todas las evidencias indican que la comunidad no demoró ni detuvo su desarrollo sucesivo corriente, pero dada la sustitución de un dominante por otro perteneciente al mismo tipo ecológico (estratega $\mathrm{S}$ ), pero más eurioico que el primero (Microcystis), el desarrollo sucesional fue desmantelado abruptamente, aunque permaneciendo en un estado estable de baja diversidad. La permanencia en este estado se ajusta a lo especificado por Sommer et al (1993) en cuanto a que una sola especie (el ecodemo introducido) contribuye con más del $80 \%$ de la biomasa total, su presencia ha persistido por largo tiempo (> 1-2 semanas) y durante este periodo la biomasa total no se ha incrementado significativamente.

Dado que la exergía acumulada en un sistema (entendida como una medida de orden) es un evaluador de su resiliencia a la perturbación, puede decirse, en concordancia con los postulados de Reynolds (1997) que, posterior al evento de introducción, ambas propiedades son altas en el sistema LPN. Un estimador de dicha magnitud es la ausencia de fluctuaciones fuertes en la densidad de $B$. braunii en el ciclo anual (Ramírez \& Corbacho, 2004) debidas a la transferencia ininterrumpida de recursos del sistema

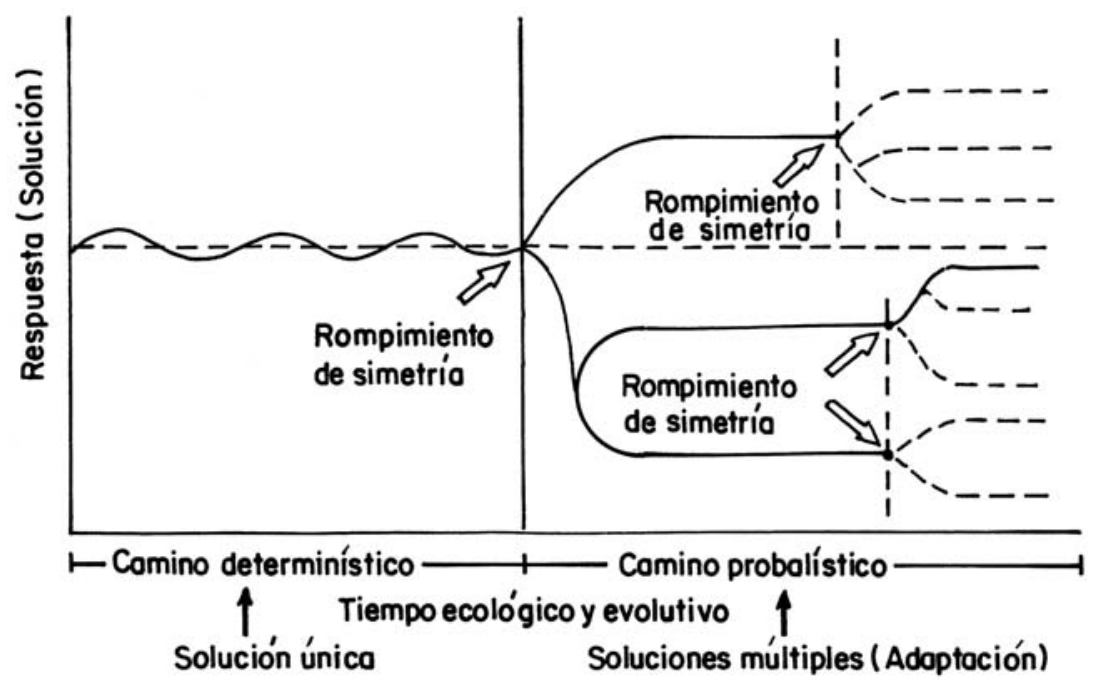

Figura 1. Representación esquemática de las posibles trayectorias seguidas por un sistema dinámico antes y después de la rotura de simetría ocasionado por un disturbio. A schematic representation of the possible trajectories followed by a dynamic system before and after the breaking of symmetry caused by a disturbance. 
a la biomasa del ecodemo dominante (Fig. 1). Esta transferencia ocurre por la condición autóctona del sistema, la cual conduce a oscilaciones mínimas representadas por las bajas variaciones temporales de los atributos físicos y químicos principales. El sistema funciona entonces como un absorbedor de choques en el que la adquisición de energía iguala a la disipación de la misma. Y aunque los organismos presenten inicialmente fluctuaciones drásticas, éstas serán cada vez menores.

Se podría pensar que una especie introducida en un sistema actúa a la manera de una piedra, creando ondas circulares que se alejan cada vez más de su centro. Estas ondas representarían la amplitud del nicho de la especie y en sus fronteras la posibilidad de defensa contra la invasión de otras especies y la posibilidad de interacción, son menores. Si no hay exclusión es porque las ondas "regresan" a su centro y expulsan a la especie invasora al igual que expulsarían a la piedra del sistema para recuperar el flujo de entropía normal al cual las especies residentes están adaptadas. En el plancton dicho símil parece no funcionar, como lo expresa la "paradoja del plancton"; sin embargo, es necesario tener en cuenta que la paradoja no predice nada respecto a la introducción de especies extrañas. La experiencia con la introducción de plantas y animales a nuevos ambientes ha demostrado que, a corto plazo, la respuesta generalmente involucra profundos cambios ecológicos, debidos a la imposibilidad de eliminar las interacciones bien sea entre partículas o ecodemos. En palabras de Prigogine (1997), si se pudieran eliminar, el universo sería isomorfo a un universo de partículas libres, y esto sería tan incoherente que no habría química, ni biología, ni culturas humanas.

Igualmente, parafraseando a Margalef (1983), un nuevo ecodemo en un sistema podría considerarse como un agente perturbador externo, un factor de estrés, de inestabilidad, de tensión que fuerza a la comunidad establecida a no seguir gobernándose por sus leyes internas y que tiende a minimizar las consecuencias de tal tensión. Si la tensión es transitoria el sistema retornará a una situación similar a aquella que ostentaba, es decir, el ecodemo será eliminado por la presión de los residentes. Si el ecodemo perturbador es mejor competidor que los locales, el sistema ecológico puede quedar acoplado funcionalmente al ecodemo forzador, separado de él por una frontera a través de la cual el intercambio no es asimétrico; esta asimetría de intercambio lo lleva a ser el dominante. Todo factor que no ha estado presente en la evolución de un ecosistema puede ser entonces un factor de tensión; este concepto incluye moléculas orgánicas inusitadas o especies de origen alóctono.

Podría también pensarse que la introducción, entendida como una perturbación externa, creó una bifurcación (posiblemente incompleta), es decir, un rompimiento del estado estable anterior. Esta nueva condición cambió la historia determinística del sistema (un ciclo de dominancias alternadas y en cierta forma predecibles, marcado por los cambios de intensidad en la mezcla) por una probabilística en la cual uno de los posibles caminos a seguir luego de la bifurcación fue la dominancia de $B$. braunii para quien, según se mencionó anteriormente, la polimixis del sistema no es un disturbio que pueda romper el estado estable y el nivel de exergía alcanzado por la comunidad (Fig. 2). Para Prigogine (1997) una propiedad de las bifurcaciones es su sensibilidad, ante pequeñas variaciones en la naturaleza del sistema, las cuales llevan a la elección preferente de una de las ramas. Este es un camino irreversible hasta que una nueva perturbación genere un nuevo nivel entrópico no menguado por las estructuras disipativas presentes en el sistema y origine un nuevo rompimiento de simetría. Por lo pronto la irreversibilidad lograda, lleva a nuevos caminos de orden, representados en la dominancia de la especie introducida.

B. braunii ha sido catalogada por Hutchinson (1967) como un ecodemo de condición eurioica, lo que explica su capacidad interactiva asimétrica y la posibilidad de ampliar las fronteras de su nicho a expensas de la exclusión de otros organismos mejor adaptados a las condiciones previas de entropía, concretamente. Los ecodemos excluidos 


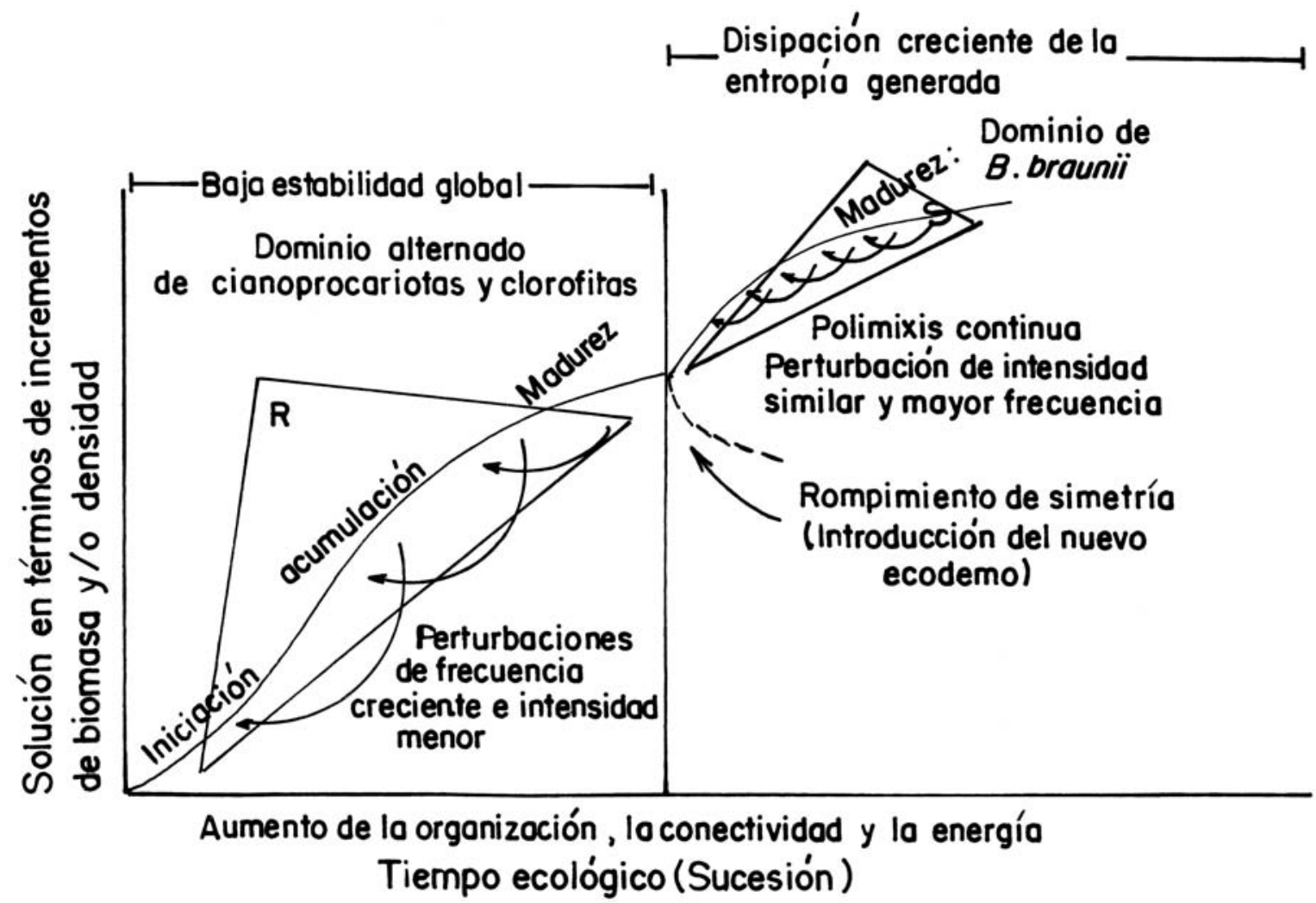

Figura 2. Representación esquemática de la solución que alcanzó el sistema Laguna del Parque Norte frente al desafío representado por la introducción de la entidad disipativa B. Braunii. Schematic representation of the solution that the Parque Norte Lagoon system reached in front of the challenge that represents the introduction of the dissipative entity, B. braunii.

no pudieron entonces disipar la nueva entrada de energía ni contrarrestar el desorden, por ello, fueron sustituidos por otros que sí lo hacen.

Según Quirós (2002) los cambios en la estructura ecosistémica son una muestras de resiliencia frente a un cambio en el ambiente de nutrientes de un lago. No es este el caso en esta investigación, pues como se mencionó, salvo el cambio en el clima óptico del lago, las demás condiciones físicas y químicas permanecieron inalteradas. Es menester recordar que para Aoki (2000), la producción de entropía de un lago por unidad de volumen del mismo no sólo aumenta con el enriquecimiento de nutrientes sino también con los incrementos de biomasa. Para este mismo autor, las cianoprocariotas y otras algas formadoras de blooms son mecanismos ecosistémicos naturales dirigidos a aliviar los cambios en el ecosistema y a maximizar las estructuras disipativas (número de organismos y biomasa) frente a gradientes externos.

La LPN posee entonces una tasa alta de producción de entropía, representada en la carga interna de nutrientes, acentuada por su condición de trampa de sedimentos y su bajo tiempo de residencia. Dicha carga funciona como las corrientes ascendentes de nutrientes en algunas regiones marinas, las cuales son disipadas por especies de peces (como la anchoveta en el Perú) y cuya dominancia disminuye drásticamente la diversidad. Algo similar se presenta en los lagos dimícticos y monomícticos, en los que la mezcla inyecta en el epilimnion una carga grande de entropía que se disipa en los blooms algales posteriores y en la disminución de la diversidad del sistema. Esta última consecuencia asegura tasas de flujo de energía relativamente bajas, que a su vez dan lugar a acumulaciones de energía capa- 
ces de ser reducidas por un invasor apropiado, a la manera de lo ocurrido con los pinzones de Darwin en las islas Galápagos y con B. braunii en el cuerpo de agua estudiado.

El nivel de dominancia presente en la LPN es debido según Johnson (1981) a la entrada alta y homogénea de energía que es el origen de un ecosistema con pocas especies, pero biomasa alta. Cobra fuerza entonces la afirmación del mismo autor en el sentido de que la dominancia que reduce el flujo de entropía en un alto grado, será más efectiva en disminuir la diversidad. La autonomía del sistema permitirá la acumulación de biomasa y la construcción de un momento significativo de amortiguación.

En cualquier sistema material aparecen interacciones y mecanismos cibernéticos y, con ellos, acumulaciones de información. Los organismos, el desarrollo de los meandros en un río, la complejidad creciente de la corteza terrestre mediante sucesivas épocas de orogénesis son dispositivos de almacenamiento de información del mismo modo que los son los sistemas genéticos. Son entonces sistemas auto-organizadores. En cualquier caso los sistemas cibernéticos que existen de un modo natural deben poseer la capacidad de persistir y, en general, a partir de la teoría cibernética se deduce que cualquier sistema que pueda adoptar diferentes estados, automáticamente permanece en, o adopta después de algún tiempo, el más estable de aquellos estados (Margalef, 1983). Esto es lo que sucedió en el sistema ecológico considerado en este trabajo.

\section{CONCLUSIONES}

En respuesta a la pregunta realizada puede decirse que la comunidad de la LPN respondió de la forma en que lo hizo porque no es resiliente ni coherente ni poseía estabilidad global. Sólo poseía estabilidad local, resiliencia y conectividad bajas. Si una comunidad no equilibrada es entonces aquella en que la abundancia de especies cambia continuamente, lo que hace impredecible su estructura, la comunidad planctónica de la LPN no lo es, al menos por perio- dos de tiempo muy grandes. Llega a serlo, cuando casos como el ocurrido desplazan el sistema hacia otro nivel de entropía no soportado por las especies dominantes residentes.

Además, la introducción del ecodemo $B$. braunii ocasionó un nuevo nivel de entropía que la comunidad no resistió y que fue generado por la interacción de la especie introducida que sí alcanzó la condición de estructura disipativa al aumentar su biomasa, adaptarse a las nuevas condiciones dada su condición eurioica; esta especie constituyó entonces una entidad disipativa que incrementó el orden de la comunidad por ser un estratega $\mathrm{S}$ de alta resistencia al cambio, mayor persistencia, pero resiliencia y variabilidad bajas.

La LPN es entonces un sistema ecológico con un dominante competitivo en el fitoplancton, siendo por tanto una comunidad controlada por la dominancia. Todo indica que, dado que el dominante fitoplanctónico permanece bajo esa condición durante casi todo el ciclo anual, la perturbación presente (encarnada en la polimixis) se ha constituido para él más en un recurso que en un disturbio.

\section{BIBLIOGRAFÍA}

ALVAREZ COBELAS, M. \& B. A. JACOBSEN 1992. Hypertrophic phytoplankton: an overview. Freshwater Forum, 2: $184-199$.

AOKI, I. 2000. Entropy and exergy principles in living systems. In: Thermodynamics and ecological modeling. S. E. Jorgensen (ed.): 167-190. Lewis Publishers, CRC Press. Boca Raton, Florida, USA.

BEGON, M.; J. L. HARPER \& C. R. TOWNSEND. 1990. Ecology. $2^{\text {nd }}$ ed. Blackwell Scientific Publications. London. 945 pp.

BROOKS, D. R. \& E. O. WILEY. 1988. Evolution as entropy. Toward a unified theory of Biology. $2^{\text {nd }} \mathrm{ed}$. Chicago: The University of Chicago Press. $451 \mathrm{pp}$.

CUNNINGHAM, W. J. 1963. The concept of stability. Am. Scientist, 51: 425-436.

HUTCHINSON, G. E. 1967. A treatise on Limnology. II. Introduction to lake biology and the limnoplankton. New York: John Wiley \& Sons. 1115 pp.

JOHNSON, L. 1981. The thermodynamic origin of ecosystems. Can. J. Fish. Aquat. Sci., 38: 571-590. 
KREBS, C. 1985. Ecología. Estudio de la distribución y la abundancia. $2^{\mathrm{a}}$ ed. México: Harla.

MARGALEF, R. 1983. Limnología. Barcelona: Ediciones Omega. 1010 pp.

NOREÑA J. F. \& J. J. RAMÍREZ. 2001. Comportamiento de la tasa de sedimentación del material sestónico en dos periodos climáticos contrastantes en un lago urbano raso: laguna del Parque Norte, Medellín (Colombia). Actual. Biol., 74: 65-74.

PIMM, S. L. 1984. The complexity and stability of ecosystems. Nature, 307: 321-326.

PRIGOGINE, I. 1997. Las leyes del caos. Barcelona: Crítica (Grijalbo Mondadori, S.A). 155 pp.

QUIRÓS, R. 2002. The nitrogen to phosphorus ratio for lakes: a cause or a consequence of aquatic biology?. In: El agua en Iberoamérica. De la limnología a la gestión en Sudamérica. Fernández, A. \& G. Chalar (eds.): 11-26. Cyted XVII-CETAFacultad de Ciencias Veterinarias, Universidad de Buenos Aires, Montevideo.

RAMÍREZ, J. J. 1987. Contribución al conocimiento de las condiciones limnológicas de la laguna del Parque Norte. Actual. Biol., 16: 12-30.

RAMÍREZ, J. J. 1994. Dinámica poblacional de dos especies de Anabaenopsis (Wolosz) V. Mill. 1923 en una laguna eutrófica tropical. Revue d'Hydrobiol. Trop., 27: 337-346.

RAMÍREZ, J. J. 2000a. Variación diurnal y estacional del contenido calórico, la estabilidad y el trabajo del viento en una laguna tropical. Acta Limnol. Bras., 12: 39-54.

RAMÍREZ, J. J. 2000b. Dinámica poblacional de Aphanizomenon aphanizomenoides en una laguna tropical rasa. Acta Limnol. Bras., 11: 27-45.
RAMÍREZ, J. J. \& A. DÍAZ. 1994. Caracterización limnológica y estructura de la comunidad fitoplanctónica de la laguna del Parque Norte en dos épocas del año en la laguna del Parque Norte. Hoenea, 21(1/2): 7-28.

RAMÍREZ, J. J. \& A. DÍAZ. 1996-1997. Fluctuación estacional del zooplancton en la Laguna del Parque Norte, Medellín, Colombia. Rev. Biol. Trop., 44(3)/45(1): 549-563.

RAMÍREZ, J. J. \& H. ALCARÁZ. 2002. Dinámica de la producción primaria fitoplanctónica en un sistema eutrófico tropical: laguna del Parque Norte, Medellín, Colombia. Caldasia, 24: 411-423.

RAMÍREZ, J. J. \& M. CORBACHO. 2004. Population dynamics of Botryococcus braunii Kützing 1849 in a shallow tropical eutrophic lake. Algol. Stud. Aceptado para publicación 115.

REYNOLDS, C. S. 1997. Vegetation processes in the pelagic: a model for ecosystem theory. Germany: Ecology Institute. $371 \mathrm{pp}$.

ROS, S. J. 1991. Mechanisms structuring fishes assemblages: are there lessons from introduced species? Env. Biol. Fishes., 30: 359-368.

SÁNCHEZ, J. I. \& J. J. RAMÍREZ. 2000. Dinámica de la comunidad zooplanctónica (excepto Protozoo) en las zonas limnética y litoral de la laguna del Parque Norte, Medellín (Colombia). Actual. Biol., 22: 65-82.

SOMMER, U.; J. PADISÁK; C. S. REYNOLDS \& P. JUHÁSZ-NAGY. 1993. Hutchinson's heritage: the diversity-disturbance relationship in phytoplankton. Hydrobiologia, 249: 1-7

ZARET, T. M. \& R. T. PAINE. 1973. Species introduction in a tropical lake. Science, 182: 449-455. 